\title{
THE TREATMENT OF GENERAL PARALYSIS BY MALARIA *
}

By W. D. NICOL, M.B., B.S.(Lond.), D.P.M., Deputy Medical Superintendent, Horton Mental Hospital.

In the first place, Sir, I wish to thank you for the honour you have accorded me in inviting me to address your society to-night.

\section{Selection of Cases}

It is now nearly four years since I became associated with Colonel James, of the Ministry of Health, at the Malarial Treatment Centre for General Paralysis of the Insane established at Horton. With regard to the selection of cases, I would point out that, as far as we are concerned, our patients are certified before they are admitted, and the diagnosis of G.P.I. does not, as a rule, present many difficulties.

All the cases treated have lumbar punctures performed beforehand, so confirming the diagnosis-the Wassermann Reaction of the cerebrospinal fluid being positivewith increased cell and protein content, and in most cases a true paretic curve is revealed in the colloidal gold test.

A matter of great importance is the physical condition of the patient. The prolonged and severe attack of malaria which is essential to the success of the treatment taxes the patient's strength considerably, and even with the greatest care involves a risk to life.

The ideal thing is to get the patient as early as possible ; advanced cases are also given the benefit of treatment, provided a thorough physical examination eliminates any organic disease (more especially of the circulatory and renal systems). On more than one occasion I have had cases sent for treatment who, on admission, have been quite unfit to infect with malaria, but after special nursing and, in some cases, open-air treatment, have recovered

* Paper read at a General Meeting of the Medical Society for the Study of Venereal Diseases on January 25th, 1929. 


\section{BRITISH JOURNAL OF VENEREAL DISEASES}

physically sufficient to warrant a course of treatment, and in some instances I am glad to say have resulted in cure. The history of seizures should not be regarded as a contra-indication to treatment.

There is one condition, however, which requires special care in managing the patient, and that is extreme obesity.

Septic foci in the teeth or alimentary system are not uncommon in G.P.I., and these should be dealt with as far as possible before treatment is commenced.

\section{Infecting Cases and Technigue}

At Horton, by arrangement between the Ministry of Health and the London County Council, a special block has been set aside, with permission of Colonel Lord, the Medical Superintendent, for the treatment of General Paralysis by Malaria.

The dormitories are mosquito proofed. A special staff of nurses is attached, who are trained in the nursing of malaria and who assist in taking observations relating to the clinical side. Two small rooms are set apart for the laboratory and entomological work, which is under the charge of Mr. Shute, a senior laboratory assistant from the Ministry of Health. Up to the last few months the work has been concerned only with a pure strain of Benign tertian and the Anopheles maculipennis, so all the cases have been infected by mosquitoes as opposed to blood inoculation.

The strain of Plasmodium vivax was first obtained in May, I925, from a patient, otherwise healthy, who had contracted malaria in Madagascar; this strain has been employed ever since. By arrangement with the Ministry of Health and the Board of Control, the strain has been available for Mental Hospitals in England and Wales; several general hospitals throughout the country also have availed themselves of the opportunity of obtaining this strain.

The mosquitoes used are collected as adults from stables and pigsties in a part of the country where they are known to be plentiful. A batch of mosquitoes so collected is infected by feeding on a patient suffering from malaria. At the end of ten to twelve days the mosquitoes become infective. They are kept in cages at a temperature of $72^{\circ} \mathrm{F}$. and transferred to small glass 


\section{TREATMENT OF PARALYSIS BY MALARIA}

feeding-bottles with wide mouths for the purpose of infecting patients. The bottles have a double layer of gauze netting over them and are applied to the patient's thigh, the mosquitoes being allowed to bite through the meshes of the gauze.

As regards blood inoculation, it is important to use syringes which are sterilised by heat only and not put in any antiseptic or spirit, because if this is done the parasites will be killed. It does not matter when the blood is drawn in relation to the fever, provided the case has been going for a few days, also that no quinine has been given : 2 to 3 c.c. blood are withdrawn from a vein, and if the patient is close at hand the blood can be injected forthwith either intravenously or intramuscularly; if the blood has to be sent away the best method is to defibrinate the blood with glass beads (five minutes should be expended in the process of defibrination); sodium citrate is not so satisfactory, and oxalated blood is definitely harmful to the parasite. In the event of having to defibrinate the blood, more blood must be withdrawn, preferably 5 to Io c.c., as a certain amount of waste occurs.

\section{The Attack and Management}

It is important that no untoward event should happen during the incubation period, which is twelve to fourteen days, as when once a patient is infected with malaria it is impossible to prevent the attack by giving quinine.

In order that all febrile manifestations of the attack may not be missed, patients should be put on a fourhourly temperature chart from the eighth day of infection. Routine daily blood examination should be commenced from the day on which the first febrile temperature is noted.

From the first rise of temperature the instructions for taking temperatures are: (I) Note the hour of the day or night at which the rise of temperature occurred on the first day; (2) On the next day, about an hour before that time, begin to take temperature every half-hour; (3) As soon as a rise above normal is observed, take the temperature every fifteen minutes and continue to do so throughout the febrile paroxysm until it has fallen to normal; (4) Then resume the four-hourly rule until the 


\section{BRITISH JOURNAL OF VENEREAL DISEASES}

next rise. This is the only plan by which a correct chart of the temperature during the attack can be obtained.

In a primary attack of benign tertian, which is nearly always quotidian in type, three stages are recognised: the initial stage, the developed stage, and the terminal stage. The initial stage begins as a gradually increasing fever which at first is sub-continuous or irregularly remittent. On the third or fourth day it becomes intermittent with the onset of the developed stage. Fig. I is an example of a usual chart.

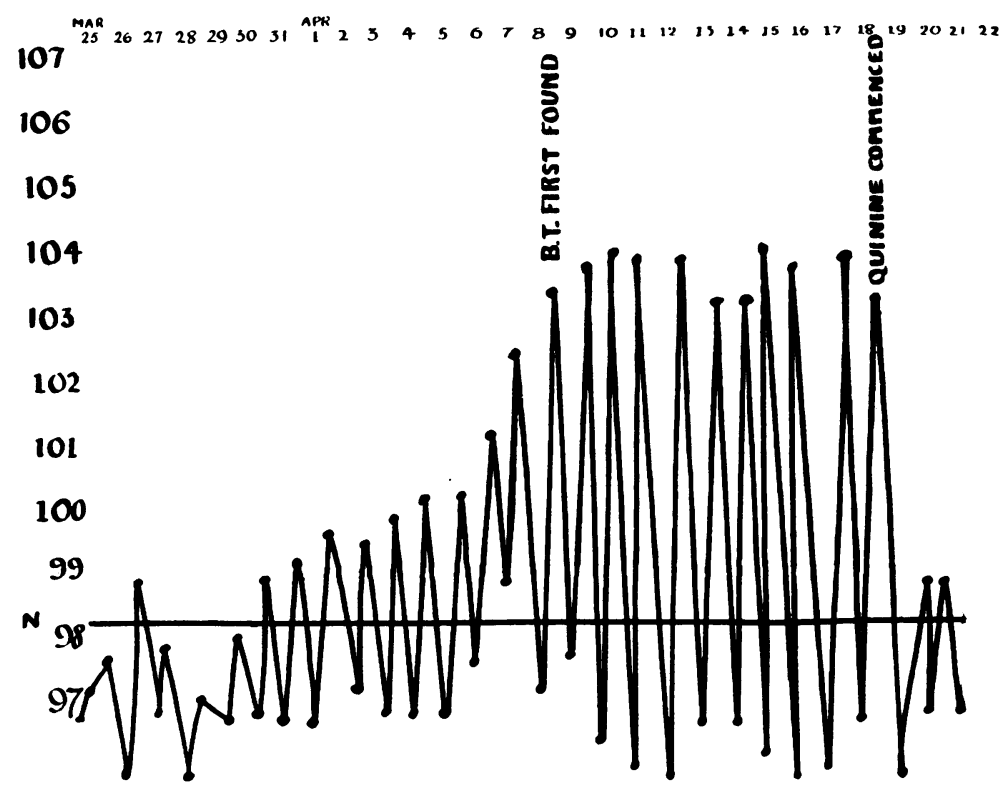

FIG. I.

The symptoms during the initial stage resemble somewhat those of a commencing attack of typhoid fever, but the spiky nature of the temperature is more apparent. There is seldom a rigor during this stage, nor will blood examination reveal parasites. It is a period of malaria which is liable to be entirely missed if the temperatures are recorded only morning and evening.

In the developed stage the temperature becomes definitely intermittent, rigors are well marked and the fever occurs daily- the temperature reaches I0 $4^{\circ}$ to IO $^{\circ}$ - parasites can be easily found in thin films of blood, and their numbers increase for the first few days until a 


\section{TREATMENT OF PARALYSIS BY MALARIA}

balance is established between the infection and the patient's resisting power, and then usually the parasites remain moderate in number.

In the management of the case during the developed stage two points are of paramount importance: (I) The temperature must be taken every fifteen minutes while the paroxysm of fever lasts, in order that appropriate measures may be taken to avoid hyperpyrexia ; (2) Blood examination should be conducted daily in order to ascertain that the number of parasites is not increasing beyond a safe limit.

With regard to the danger of hyperpyrexia, it is our practice at Horton to cold sponge at $105^{\circ}$, and to repeat it as often as may be necessary to keep it at or below $105^{\circ}$

Temperatures During the Malarial Paroxysm. Duration i4 Hours
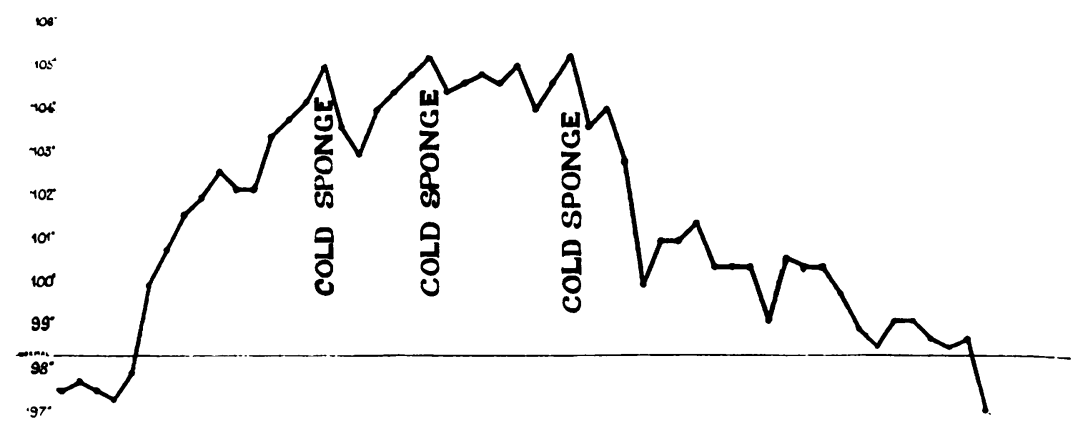

FIG. 2.

while the paroxysm lasts. This is illustrated in Fig. 2, which shows the duration of the paroxysm (usually ten to twelve hours) and the importance of taking temperatures frequently.

As regards the parasite infection, it is the practice to examine a blood film stained with Leishmann daily. Using one-twelfth oil immersion and a No. 2 eye-piece twenty-five fields are passed under review. If thirty-five or more parasites are found (i.e., more than one parasite in every field) it is considered an indication that the fever should be stopped.

Here I come to what I consider the most important procedure in the whole of the treatment, and that is the temporary interruption of the course of fever. I do not think too great stress can be laid on this point, as I feel certain it is one of the biggest factors in reducing the 


\section{BRITISH JOURNAL OF VENEREAL DISEASES}

mortality from malaria, which during the first two months following treatment is still perhaps rather high. This is effected by giving one dose of quinine, 5 gr., and if necessary repeating the same the next day; the result usually is cessation of fever and almost complete disappearance of parasites from the peripheral blood, to be followed in from ten to twenty days by a recrudescence (see Fig. 3). The advantages are, the strain of malaria is. not lost, an important point if the patient has only had three to four peaks of fever ; during this temporary interval the patient's physical condition improves and the recrudescence, when it does occur, is not so severe as. the original attack.

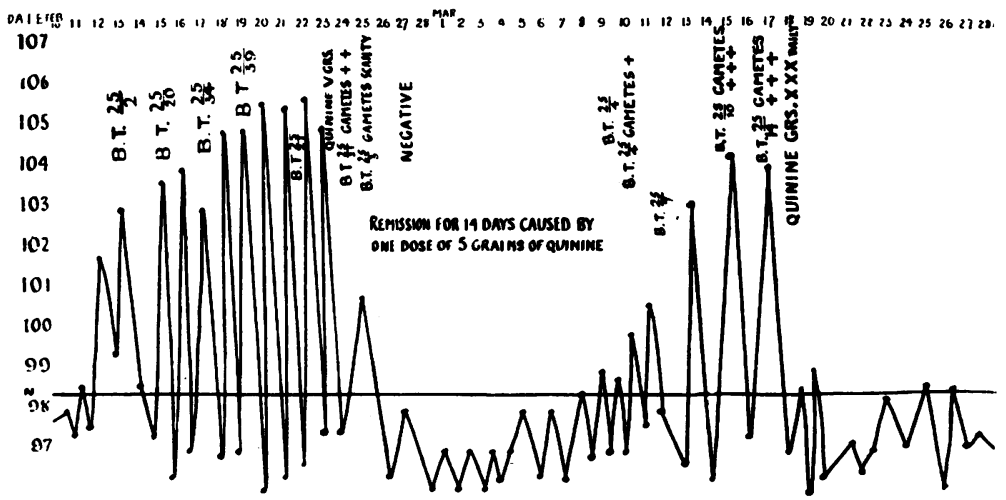

FIG. 3 .

The indications for temporary interruption, besides a heavy parasite count, may be taken as persistent vomiting, faintness or collapse during the paroxysm, cyanosis, seizures, undue restlessness, persistent high pulse rate, and the earliest sign of jaundice. Also another point is worth watching, and that is the failure of the temperature to fall right down to subnormal in between the paroxysms.

In primary attacks the terminal stage is not often observed, as the fever is generally stopped beforehand. When it does occur it is characterised by the quotidian fever becoming tertian and by a gradually diminishing. severity of the attacks.

With regard to the management during the paroxysm, the patient must be kept warm between blankets, hot water bottles must be put in bed, hot broth or fresh tea 


\section{TREATMENT OF PARALYSIS BY MALARIA}

should be given to patients directly they begin to feel cold. The instructions regarding the taking of temperatures have already been mentioned. During the hot stage the patient should be made to drink as much fluid as possible-barley water, soda or alkaline drinks. For nausea or vomiting a dose of gentian with soda is useful. It is very important to promote sweating, as the patient gets almost immediate relief.

In between the paroxysms the patient may have ordinary nourishing diet; constipation is the rule, and generally daily enemata are required, in addition to mild aperients. The urine should be examined daily, albuminuria is not infrequent, but provided the amount is not great I do not attach any importance to its presence. The heart and pulse rate must be carefully watched and, if necessary, digitalis should be given. In many cases herpes labialis is common.

\section{Treatment of the Attack}

Ten to twelve paroxysms of fever should be aimed at, after which the attack can be terminated by giving quinine. From the experience at Horton it is found that small doses of quinine are just as efficacious as the large doses, and it has been our practice to give quinine bihydrochloride, 5 grs., once daily for fifteen days. The fever subsides within forty-eight hours and the blood is free from parasites after the third day.

Here again I would like to lay stress on the danger of giving too much quinine in those cases which become gravely ill either from toxæmia or a heavy parasite infection or commencing jaundice. We have found that the best means of dealing with cases such as these is to give the one dose of quinine, 5 grs., and allow the patient to settle for a few days, during which time the general condition will almost invariably improve, and then commence the full course of quinine.

Patients are generally exhausted and anæmic after a prolonged course, and the treatment during convalescence should be supplemented by an iron tonic. A small quantity of stout should also be allowed. As an aid to the cure of G.P.I. many physicians supplement the course of malaria by giving an anti-syphilitic course of one of the organic arsenical compounds. 


\section{BRITISH JOURNAL OF VENEREAL DISEASES}

\section{RELAPSES}

It has been found that 60 to 70 per cent. of the mosquito inoculated cases relapse with malaria. Usually they are long relapses, occurring six to seven months after the primary attack. The fever is generally mild in character, of tertian type, and the best course to adopt is to allow the relapse to run, when a spontaneous recovery will occur after about seven to ten days, and this can be followed up by giving a full course of quinine. I have not known a case so treated to give any trouble with relapses after one year following the primary attack.

\section{Quartan Malaria}

I would like to say a few words about treatment of General Paralysis, not by benign tertian, but by quartan malaria. Quartan malaria is the mildest of the three types of malaria, and is characterised by a paroxysm of fever occurring only every fourth day instead of every third day as in true benign tertian malaria and intermittent in subtertian.

The rigors are less severe and sometimes absent altogether. The paroxysm of fever, though lasting longer, does not affect the patient to the same extent. The interval of two days without fever allows the patient more time for recovery than in benign tertian. During the last three months a strain has been employed on some patients, and it has been found that the severity of the malaria is much less, despite the fact that the temperature will reach $105^{\circ}$ (as is the case with benign tertian), and cold sponging has not been found necessary; also the patients can readily stand quartan fever lasting six and seven weeks, which includes twenty peaks of fever (as against ten to twelve in benign tertian), and even then the patient's general condition will remain quite good, anæmia being hardly noticeable.

The fever can be easily controlled by quinine. It is too early to speak of results, but there are some advantages which are apparent :

(I) Patients who might be given benign tertian with considerable risk on account of their general physical condition will tolerate quartan well; (2) the nursing requirements are less arduous and the case can be more easily looked after; (3) here I come to the question of 


\section{TREATMENT OF PARALYSIS BY MALARIA}

immunity. It is generally recognised that many patients acquire immunity to benign tertian, and in many cases, though the physical condition has improved following an attack of primary benign tertian, the mental condition has remained unchanged. This type of case should be given the benefit of a second course of treatment. In those cases which prove to be immune to a second infection of benign tertian, quartan has been found efficacious in affording a second course; (4) lastly, though not common in this country, but from the literature quite common in America, I feel that quartan malaria affords the only solution for giving those patients malarial treatment who subsequently develop G.P.I. after spending many years in the tropics. It is found that many have acquired immunity to malaria, and as quartan is the rarest of all three types of malaria, it follows that most cases which fail to respond to treatment by benign tertian will take quartan readily. This has actually been worked out by us at Horton.

The strain of quartan employed was obtained from Professor Kirschbaum, of Hamburg. He has been treating cases of G.P.I. with quartan during the last seven years and speaks very highly of the results, claiming a remission rate as high as 50 per cent.

\section{RESULTS}

I propose to touch very briefly on the results of malaria treatment. The only statistics available on a large scale are those of the public mental hospitals in England and Wales, and it is generally agreed that, roughly speaking, the cases can be divided into thirds-one-third improved physically and mentally and able to be discharged ; onethird improved physically, but mentally (though slightly improved) institutionalised; one-third no change, with generally progressive deterioration.

It appears that the results of the mosquito inoculated cases are more favourable than those of the blood inoculated cases. Is this not due, perhaps, to the fact that a large proportion of the mosquito inoculated cases relapse, thus demonstrating that there must be some continuous process at work whereby the malaria toxin is acting on the diseased brain of the general paralytic ? The blood inoculated cases receive their quinine at the termination 


\section{BRITISH JOURNAL OF VENEREAL DISEASES}

of the primary attack and there is an end of the malaria, for relapses in blood inoculated cases are unknown.

Analysing the first Ioo cases at Horton, I subdivided them into different clinical types of G.P.I.-manic, depressed, grandiose, simple dementing, paranoid, and stuporose. It is very striking how the manic and grandiose types respond far better than the other types, there being 50 per cent. of cases remitting, while the simple dementing type (unfortunately the biggest group) yields only I2 per cent. remissions. The stuporose type is rare ; I have only seen two. The paranoid type, also a small group, appears to hold out a very poor prognosis.

\section{DiAgNosis}

Lastly, may I be permitted to say a word on the diagnosis of G.P.I. At Horton and other mental hospitals the cases treated are already certified, and consequently there has been in many cases regrettable delay before treatment could be commenced. There is no doubt that the sooner the case is diagnosed the better the prospect of recovery. Last year I was fortunate in seeing cases not only in mental hospitals, but also many uncertified cases in general hospitals. From observation of the last group of cases I cannot help feeling that there is an early stage in which both mental and physical signs are practically non-existent, but there is the all-important factor of a positive Wassermann in the cerebrospinal fluid.

Bunker,* in America, has analysed the early mental symptoms, which he enumerates in order of frequency : Emotional irritability, with the patient restless and fidgety; abnormal quietness, the patient being preoccupied, listless and apathetic ; loss of weight ; forgetfulness ; increased tendency to sleep; defective judgment. All these symptoms are extremely vague and much depends on the individual skill of the clinician, but patients between the ages of thirty to fifty, who complain of indefinite neurotic symptoms, should always be examined for general paralysis. The secret of success of malaria therapy lies in the early diagnosis.

* American Journal of Medical Science, 1926, 386. 